\section{Equidade no acesso ao rastreamento mamográfico do câncer de mama com intervenção de mamógrafo móvel no sul do Rio Grande do Sul, Brasil}

\author{
Equity in access to breast cancer screening in a \\ mobile mammography program in southern \\ Rio Grande do Sul State, Brazil
}

Décio Valente Renck 1

Fernando Barros 1

Marlos Rodrigues Domingues 2

Maria Cristina Gonzalez 1

Marcelo Leal Sclowitz 3

Eduardo Lucia Caputo 2

Laura de Moraes Gomes 4

\author{
1 Programa de Pós-graduação \\ em Saúde e Comportamento, \\ Universidade Católica de \\ Pelotas, Pelotas, Brasil. \\ 2 Escola Superior \\ de Educação Física, \\ Universidade Federal de \\ Pelotas, Pelotas, Brasil. \\ ${ }_{3}$ Faculdade de Medicina, \\ Universidade Federal de \\ Pelotas, Pelotas, Brasil. \\ ${ }^{4}$ Departamento de \\ Radiologia, Hospital Santa \\ Casa de Misericórdia de \\ Pelotas, Pelotas, Brasil. \\ Correspondência \\ M. L. Sclowitz \\ Departamento Materno- \\ Infantil, Faculdade de \\ Medicina, Universidade \\ Federal de Pelotas. \\ Rua Barão de Santa Tecla \\ 583, sala 604, Pelotas, RS \\ 96010-140, Brasil. \\ sclowitzml@terra.com.br
}

\begin{abstract}
This study aimed to evaluate a program for the prevention and early diagnosis of breast cancer in a mobile mammography unit that visited several cities in Rio Grande do Sul, the southernmost State in Brazil, where the test was not available through routine services. The authors compared the characteristics of these patients with a group of women referred for mammography in the city of Pelotas, where such equipment is widely available. The mobile unit examined 8,607 women residing in 33 municipalities. The study identified 37 cases of breast cancer, or 4.7 cases per 1,000 tests. In Pelotas, 1,312 patients underwent mammography, and breast cancer prevalence was 6.9 cases per 1,000 tests. There were no statistical differences between the two prevalence rates, even after adjusting for potential confounders. The proposed intervention demonstrates that the availability of tests is needed for prevention and early diagnosis of breast cancer, determining the identification of cases when diagnosis would have been delayed without the mobile unit.
\end{abstract}

Mammography; Biomedical Technology; Breast Neoplasms

\section{Resumo}

O objetivo foi avaliar um programa de prevenção e diagnóstico precoce de câncer de mama, realizado com uma unidade móvel de mamografia que visitou municípios da região sul do Rio Grande do Sul, Brasil, onde não havia um aparelho de mamografia disponível. As características das mulheres avaliadas pelo mamomóvel foram comparadas com as de mulheres encaminhadas para exame mamográfico na cidade de Pelotas. Com a unidade móvel foram examinadas 8.607 mulheres residentes em 33 municípios da zona sul, e foram identificados 37 casos de câncer de mama (4,7 casos por mil exames realizados). Em Pelotas, foram examinadas 1.312 pacientes, e a prevalência de câncer de mama foi de 6,9 casos por mil exames. A prevalência de casos positivos foi estatisticamente semelhante nos dois grupos, mesmo após o ajuste para possíveis fatores de confusão. A intervenção proposta demonstra que a disponibilização de exames é necessária na prevenção e diagnóstico precoce do câncer de mama, determinando a identificação de casos cujos diagnósticos teriam sido retardados se esta unidade não estivesse presente.

Mamografia; Tecnologia Biomédica; Neoplasias da Mama 


\section{Introdução}

A inexistência de recursos diagnósticos na área de moradia é uma barreira importante a atividades de prevenção e diagnóstico precoce de doenças graves, como as neoplasias. Nos locais onde existem aparelhos necessários para o atendimento da população ocorre falha na distribuição dos mesmos, fazendo com que indivíduos com melhores condições socioeconômicas tenham acesso mais facilitado a testes de rastreamento dessas doenças 1,2 .

O câncer de mama é o tumor que ocorre com maior frequência na população feminina ${ }^{3}$. Até os 40 anos de idade, esse tipo de câncer ocorre de forma rara, $85 \%$ dos casos acontecem após os 40 anos 4 . No Brasil, são 52 novos casos de câncer de mama por ano a cada grupo de $100 \mathrm{mil} \mathrm{mu-}$ lheres. No Estado do Rio Grande do Sul esse número quase dobra, são 81 casos anuais em cada grupo de 100 mil mulheres. A cada 50 mil novos casos surgidos no Brasil, 5 mil são no Rio Grande do Sul. O índice de cura esperado é de $70 \%$ nos casos de câncer de mama, mas em nosso estado o índice de cura fica abaixo dos $50 \%$. Esse baixo índice de cura evidenciado deve-se, em parte, à descoberta tardia da doença 5 .

Histórico familiar, menarca precoce, menopausa tardia, idade avançada para a primeira gravidez, nuliparidade, não amamentar ao peito, uso de contraceptivo oral, terapia de reposição hormonal e exposições à radiação ionizante, assim como hábitos de estilo de vida não saudáveis são fatores de risco para a ocorrência do câncer de mama $6,7,8$.

A detecção precoce do tumor pode se dar por meio das seguintes medidas preventivas: autoexame mensal das mamas; exame clínico anual das mamas realizado por profissional de saúde; $\mathrm{e}$ a mamografia, que consiste em um exame radiológico das mamas 5 .

Renda familiar, escolaridade e local de moradia (zona rural ou urbana), apresentam associação com a realização de mamografia. Existe um aumento da realização de mamografia em indivíduos com alta renda familiar, boa escolaridade e que residem na zona urbana 9 .

Este estudo descreve a utilização de uma unidade móvel com mamógrafo em municípios de uma região do Rio Grande do Sul onde este equipamento não estava disponível para a população. O objetivo do trabalho da unidade móvel foi oferecer alternativas de prevenção e diagnóstico precoce do câncer de mama à comunidade feminina da zona sul do Rio Grande do Sul, das zonas rural e urbana, com relação aos cuidados da saúde mamária, proporcionando às participantes exames em local onde este serviço não é ofertado ou sua oferta se dá em local muito distante.

\section{Materiais e métodos}

Neste trabalho apresentamos as características sociodemográficas e reprodutivas, assim como a prevalência de diagnóstico de câncer de mama nas mulheres que realizaram exame mamográfico no mamomóvel, por não terem acesso ao aparelho na sua região, tendo sido encaminhadas para exame após a triagem realizada por mastologistas uma semana antes da visita desta unidade. Essa população foi comparada, para fins de melhor contextualizar esses achados, com outra amostra de mulheres residentes na área urbana de Pelotas, que foram encaminhadas para exame por profissionais de saúde da rede pública ou privada.

Foram examinados dois grupos de mulheres que apresentavam as seguintes características: a) Mulheres que foram recrutadas por meio de campanha envolvendo a Universidade Federal de Pelotas (UFPel), a Associação Voluntárias da Mama (Mama Vida) e a Fundação de Apoio Universitário (FAU). Foram realizados exames de mamografia em mulheres residentes nas áreas urbana e rural de 33 municípios da zona sul do Estado do Rio Grande do Sul, cujas distâncias dos serviços especializados mais próximos variam de 18 a 260 quilômetros. Esses municípios, que estão listados na Tabela 1, em sua maioria não possuíam equipamento mamográfico, e as mulheres examinadas foram previamente triadas por médicos mastologistas que visitaram o município uma semana antes da chegada do mamomóvel. As razões para o encaminhamento foram idade superior a 40 anos, ou, para as mulheres mais jovens, a existência de algum fator de risco, como história familiar de câncer de mama ou achados clínicos suspeitos.

b) Mulheres residentes na área urbana de Pelotas, que foram encaminhadas de ambulatórios municipais e clínicas privadas, também por apresentarem alguma indicação, tal como as do grupo antes descrito.

Todos os exames, para os dois grupos de mulheres, foram realizados pelo MamomóvelCaminhão Baú equipado com mamógrafo, unidade reveladora de raios-X e sala para exames. As participantes responderam a um questionário contendo informações sociodemográficas e de saúde.

O projeto contou com equipe multidisciplinar, garantindo a integridade da atenção às mulheres, qualidade de rastreamento, diagnóstico, tratamento e cuidados paliativos. Foram obser- 
Municípios que participaram do estudo. Rio Grande do Sul, Brasil.

\begin{tabular}{|c|c|c|}
\hline Municípios participantes & População (habitantes) & Distância até a cidade sede (km) \\
\hline Amaral Ferrador & 5.629 & 110 \\
\hline Arambaré & 4.476 & 185 \\
\hline Arroio do Padre & 2739 & 18 \\
\hline Arroio Grande & 19.780 & 96 \\
\hline Bagé & 121.299 & 195 \\
\hline Barra do Ribeiro & 12.532 & 250 \\
\hline Caçapava do Sul & 34.659 & 235 \\
\hline Camaquã & 63.735 & 139 \\
\hline Candiota & 9.601 & 142 \\
\hline Canguçu & 52.124 & 60 \\
\hline Capão do Leão & 26.740 & 18 \\
\hline Cerrito & 6.929 & 70 \\
\hline Chuí & 6.386 & 260 \\
\hline Chuvisca & 4.622 & 171 \\
\hline Cristal & 6.981 & 100 \\
\hline Dom Feliciano & 14.380 & 185 \\
\hline Eldorado do Sul & 33.261 & 250 \\
\hline Encruzilhada do Sul & 25.132 & 180 \\
\hline Herval & 7.516 & 154 \\
\hline Hulha Negra & 4.102 & 165 \\
\hline Jaguarão & 31.558 & 130 \\
\hline Morro Redondo & 5.953 & 40 \\
\hline Pedras Altas & 2.746 & 150 \\
\hline Pedro Osório & 8.210 & 70 \\
\hline Pelotas & 342.513 & - \\
\hline Pinheiro Machado & 14.467 & 100 \\
\hline Piratini & 20.516 & 80 \\
\hline Rio Grande & 195.392 & 60 \\
\hline Santana da Boa Vista & 8.754 & 110 \\
\hline São José do Norte & 24.877 & 80 \\
\hline São Lourenço do Sul & 45.210 & 60 \\
\hline Santa Vitória do Palmar & 34.597 & 250 \\
\hline Tapes & 17.704 & 195 \\
\hline Turuçu & 3.929 & 40 \\
\hline Total (34 municípios) & 1.219 .049 & - \\
\hline
\end{tabular}

vados todos os princípios do Sistema Único de Saúde (SUS), com destaque para o acesso universal e gratuito.

Realizou-se uma parceria com as prefeituras dos municípios, que por meio de suas secretarias municipais de saúde disponibilizaram as instalações necessárias para a realização dos mutirões de exames clínicos, local para a instalação da unidade móvel com a devida ligação na rede elétrica, disponibilização de pessoal para o auxílio no trabalho (preenchimento de fichas, or- ganização dos atendimentos etc.), transporte de pacientes que necessitassem de tratamento no Hospital Escola UFPel/FAU em Pelotas, entrega dos exames realizados em seus domicílios, entre outros. O caminhão, equipado com mamógrafo, estava dentro das normas da vigilância sanitária e de radioproteção.

As características estudadas foram: idade, estado menstrual/menopausal, tabagismo, número de filhos, histórico familiar, amamentação, realização de mamografia anteriormente, auto- 
exame e os achados da mamografia realizada. O diagnóstico foi realizado com base nas categorias propostas pela classificação BI-RADS (Breast Imaging-Reporting and Data System. http://www. birads.at/).

Os dados foram digitados em planilha Excel (Microsoft Corp., Estados Unidos) e posteriormente transferidos para o programa Stata (Stata Corp., College Station, Estados Unidos) para a realização das análises estatísticas. Esses dados estão apresentados por meio de médias e seus respectivos desvios padrão para variáveis contínuas, numéricas, e em proporções, quanto às variáveis categóricas. Foi utilizado o teste t de Student para a comparação entre médias, e o teste do qui-quadrado para a comparação entre proporções. Para verificar a associação entre as variáveis independentes e os achados da mamografia fez-se uso de regressão logística. No modelo de regressão utilizou-se o diagnóstico do exame mamográfico como variável dependente, e as possíveis variáveis de confundimento foram aquelas que apresentaram um valor de $\mathrm{p}$ abaixo de 0,20 na análise bruta.

\section{Resultados}

Ao todo foram realizadas mamografias em 9.919 mulheres, sendo 8.607 nos locais visitados pelo Mamomóvel e 1.312 exames realizados em Pelotas, no Hospital Escola da UFPel/FAU. As principais características das mulheres examinadas nesses dois grupos estão descritas na Tabela 2. As mulheres dos dois grupos tinham o mesmo padrão etário - média de 51 anos - e tiveram a menarca com a mesma média de idade. O histórico reprodutivo (número de filhos), o histórico familiar de câncer de mama e a prática de autoexame das mamas foram também semelhantes nos dois grupos. Entretanto, esses diferiram em relação à prevalência de menopausa - maior no grupo do Mamomóvel (43\%) em relação ao grupo de Pelotas (33\%), assim como em relação ao tabagismo e histórico de amamentação (um pouco mais elevados entre as mulheres de Pelotas), e realização de mamografia anteriormente - bem mais elevada no grupo de Pelotas (73,6\%) do que no grupo do Mamomóvel (52,1\%).

A Tabela 3 mostra a prevalência de câncer de mama conforme as variáveis com plausibilidade biológica de associação com a doença, como idade, ocorrência de menopausa, tabagismo, histórico de amamentação, histórico familiar de câncer de mama e realização prévia de exame mamográfico. A tabela mostra também as razões de prevalência, com intervalos de confiança (IC95\%), e os respectivos valores de p. Iden- tificou-se 46 casos de câncer de mama entre as 9.919 mulheres examinadas, correspondendo a 4,6 casos por cada mil mulheres. A única variável significativamente associada com a doença foi a idade, com uma razão de prevalência 3,3 vezes maior para as mulheres acima de 58 anos.

A Tabela 4 mostra a prevalência de câncer de mama de acordo com o local de realização do exame. Entre as 1.312 mulheres que fizeram o exame no Hospital Escola da UFPel/FAU foram identificados 9 casos (6,9 casos por mil mulheres), enquanto que entre as 8.607 avaliadas no Mamomóvel foram identificados 37 casos de câncer de mama (4,3 casos por mil mulheres). A razão de odds de câncer de mama entre as mulheres avaliadas no Hospital Escola da UFPel/ FAU, em relação àquelas examinadas no Mamomóvel, foi de 1,60 (IC95\%: 0,77-3,32). Portanto, não houve diferença estatisticamente significativa entre a prevalência do câncer de mama nos dois grupos de mulheres estudadas. Os valores da razão de odds não foram modificados quando foram adicionadas, em um modelo de regressão logística, as variáveis anteriormente descritas como possíveis fatores de confundimento para esta associação: idade da mulher, menopausa, tabagismo, histórico de amamentação, realização prévia de mamografia e histórico familiar de câncer de mama. A razão de odds após o ajuste pouco modificou (1,56; IC95\%: 0,73-3,31).

A associação entre a distância do mamógrafo e a realização prévia de mamografia também foi testada. Os municípios estudados foram divididos em dois grupos conforme a distância em relação ao equipamento de mamografia mais próximo: um grupo dos municípios distantes mais de $250 \mathrm{~km}$, e outro grupo daqueles situados a menos de $250 \mathrm{~km}$. Nos municípios mais distantes a prevalência de mulheres que não haviam realizado mamografia prévia foi de 51,3\% contra $44,2 \%$ daquelas dos municípios mais próximos ( $\mathrm{p}<0,001$ ). Para todas as mulheres estudadas, $45,1 \%$ nunca tinham feito mamografia antes.

\section{Discussão}

No Brasil e no Rio Grande do Sul são identificados, respectivamente, 52 e 81 casos de câncer de mama por grupos de 100 mil mulheres, contando apenas os casos identificados em hospitais e clínicas com serviços especializados de mamografia ${ }^{10}$. Nossos dados indicam uma prevalência de 4,6 casos a cada mil mulheres (ou 460 casos por 100 mil mulheres), valores muito superiores aos descritos para o país e o estado. É possível que o rastreamento prévio realizado por médicos especialistas, e o encaminhamento para exame 
Tabela 2

Características da amostra. Rio Grande do Sul, Brasil.

\begin{tabular}{|c|c|c|c|c|}
\hline \multirow[t]{2}{*}{ Variável } & \multicolumn{2}{|c|}{ Local de realização do exame } & \multirow[t]{2}{*}{ Total $(\mathrm{N}=9.919)$} & \multirow[t]{2}{*}{ Valor de $p$} \\
\hline & Mamomóvel ( $n=8.607)$ & FAU $(n=1.312)$ & & \\
\hline \multicolumn{5}{|l|}{ Idade (anos) } \\
\hline$<40$ & $7,8 \%$ & $9,3 \%$ & $8,0 \%$ & 0,140 * \\
\hline$>40$ & $82,2 \%$ & $90,7 \%$ & $92,0 \%$ & \\
\hline Idade (anos) [média e DP] & $51,2(10.4)$ & $51,4(10,4)$ & $51,2(9,8)$ & 0,368 * \\
\hline Idade menarca (anos) [média e DP] & $13,0(1,6)$ & $13,0(1,7)$ & $13,0(1,6)$ & 0,170 * \\
\hline \multicolumn{5}{|l|}{ Ainda menstrua } \\
\hline $\operatorname{Sim}$ & $44,2 \%$ & $41,8 \%$ & $43,9 \%$ & 0,108 * \\
\hline Não & $55,8 \%$ & $58,2 \%$ & $56,1 \%$ & \\
\hline \multicolumn{5}{|l|}{ Está na menopausa } \\
\hline Sim & $43,1 \%$ & $33,2 \%$ & $41,8 \%$ & 0,001 * \\
\hline Não & $56,9 \%$ & $66,8 \%$ & $58,2 \%$ & \\
\hline \multicolumn{5}{|l|}{ Fuma } \\
\hline Sim & $18,9 \%$ & $22,3 \%$ & $19,3 \%$ & 0,004 * \\
\hline Não & $81,1 \%$ & $77,7 \%$ & $80,7 \%$ & \\
\hline \multicolumn{5}{|l|}{ Número de filhos } \\
\hline Nenhum & $7,0 \%$ & $7,3 \%$ & $7,0 \%$ & 0,906 * \\
\hline 1 & $16,1 \%$ & $16,3 \%$ & $16,1 \%$ & \\
\hline 2 & $30,6 \%$ & $31,1 \%$ & $30,7 \%$ & \\
\hline $3 \mathrm{ou}+$ & $46,3 \%$ & $45,3 \%$ & $46,2 \%$ & \\
\hline \multicolumn{5}{|l|}{ Amamentou } \\
\hline Sim & $81,8 \%$ & $85,1 \%$ & $82,2 \%$ & 0,003 * \\
\hline Não & $18,2 \%$ & $14,9 \%$ & $17,8 \%$ & \\
\hline \multicolumn{5}{|l|}{ Histórico familiar de câncer mama } \\
\hline $\operatorname{Sim}$ & $22,0 \%$ & $21,4 \%$ & $21,9 \%$ & 0,533 * \\
\hline Não & $78,0 \%$ & $78,6 \%$ & $78,1 \%$ & \\
\hline \multicolumn{5}{|l|}{ Fez autoexame } \\
\hline $\operatorname{Sim}$ & $80,7 \%$ & $81,4 \%$ & $80,8 \%$ & 0,533 * \\
\hline Não & $19,3 \%$ & $18,6 \%$ & $19,2 \%$ & \\
\hline \multicolumn{5}{|l|}{ Já fez mamografia } \\
\hline Sim & $52,1 \%$ & $73,6 \%$ & $54,9 \%$ & 0,001 * \\
\hline Não & $47,9 \%$ & $26,4 \%$ & $45,1 \%$ & \\
\hline
\end{tabular}

DP: desvio-padrão; FAU: Fundação de Apoio Universitário.

* Teste do qui-quadrado.

somente do grupo de mulheres com algum fator de risco, tenham sido responsáveis pela maior prevalência de câncer de mama em nossa amostra. Tais achados demonstram a importância do rastreamento ativo do câncer de mama, como forma de diagnóstico precoce, prevenção da doença e redução dos índices de mortalidade.

A média de idade das mulheres participantes foi de 51,2 anos, sendo que as com idades acima de 40 anos representavam $92 \%$ da amostra. No Rio de Janeiro, Godinho \& Koch 11 relataram uma média de idade de 49,1 anos e maior prevalência de mamografia, tanto em hospitais públicos como nos privados, em mulheres com idade superior a 40 anos, representando $94 \%$ no serviço público e $82,2 \%$ no privado. O aumento do rastreamento, realizado de forma regular, pode reduzir entre $30 \%$ e $40 \%$ a mortalidade em mulheres nessa faixa etária, principalmente após os 50 anos 12 .

$\mathrm{O}$ risco de câncer de mama aumenta quando há história familiar da doença ${ }^{8}$, e em nossa amostra $22 \%$ das mulheres relataram ter algum familiar com este tipo de câncer. Outro estudo carioca realizado em 200613 encontrou que 29,1\% das mulheres relataram tal fato. Em pesquisa 
Tabela 3

Prevalências e razões de prevalência (RP), com intervalos de 95\% de confiança (IC95\%), de câncer de mama conforme variáveis com plausibilidade biológica de associação. Rio Grande do Sul, Brasil.

\begin{tabular}{|c|c|c|c|c|c|}
\hline Variável & Casos & $\mathbf{n}$ & Casos/ 1.000 exames & RP (IC95\%) & Valor de $p$ \\
\hline Menopausa & & & & & 0,81 \\
\hline Sim & 20 & 4.141 & 5 & $1,00(0,59-1,92)$ & \\
\hline Não & 26 & 5.771 & 5 & 1,00 & \\
\hline Idade (anos) & & & & & $<0,001$ \\
\hline$\leq 44$ & 8 & 2.788 & 3 & 1,00 & \\
\hline $45-50$ & 8 & 2.387 & 3 & $1,00(0,43-3,11)$ & \\
\hline $51-58$ & 8 & 2.468 & 3 & $1,00(0,42-3,06)$ & \\
\hline$>59$ & 22 & 2.276 & 10 & $3,33(1,50-7,63)$ & \\
\hline Tabagismo & & & & & 0,14 \\
\hline Sim & 5 & 1.916 & 3 & $0,60(0,20-1,20)$ & \\
\hline Não & 41 & 7.999 & 5 & 1,00 & \\
\hline Amamentação & & & & & 0,15 \\
\hline Sim & 36 & 8.154 & 4 & 1,00 & \\
\hline Não & 9 & 1.152 & 8 & $2,00(0,85-3,69)$ & \\
\hline Mamografia prévia & & & & & 0,93 \\
\hline Sim & 25 & 5.448 & 5 & 1,00 & \\
\hline Não & 21 & 4.468 & 5 & $1,00(0,57-1,83)$ & \\
\hline Histórico familiar & & & & & 0,73 \\
\hline Sim & 9 & 2.170 & 4 & $1,25(0,41-1,80)$ & \\
\hline Não & 37 & 7.745 & 5 & 1,00 & \\
\hline Total & 46 & 9.919 & 5 & & \\
\hline
\end{tabular}

Tabela 4

Prevalências e razões de odds bruta e ajustada de câncer de mama, conforme local de realização do exame. Rio Grande do Sul, Brasil.

\begin{tabular}{|c|c|c|c|c|}
\hline \multirow[t]{2}{*}{ Variável } & \multicolumn{2}{|c|}{ Local de realização do exame } & \multirow[t]{2}{*}{ Total $(\mathrm{N}=9.919)$} & \multirow[t]{2}{*}{ Valor de $p$} \\
\hline & Mamomóvel $(n=8.607)$ & $\mathrm{FAU}(n=1.312)$ & & \\
\hline Casos de câncer de mama & 37 & 9 & 46 & \\
\hline Casos por 1.000 exames & 4,3 & 6,9 & 4,6 & 0,203 \\
\hline Razão de odds bruta & 1,0 & $1,60(0,77-3,32)$ & 9.919 & 0,208 \\
\hline Razão de odds ajustada * & 1,0 & $1,56(0,73-3,31)$ & 9.902 & 0,234 \\
\hline
\end{tabular}

FAU: Fundação de Apoio Universitário.

* Ajustada para idade da mulher, menopausa, tabagismo, história de amamentação, realização prévia de mamografia.

realizada na cidade de Pelotas, a prevalência de história familiar de câncer de mama foi de $15,2 \% 14$. Como esperado, mulheres que apresentam histórico familiar de câncer de mama demonstram maior probabilidade de realizar exame de mamografia, quando comparadas a mulheres que não apresentam este fator de risco 15,16.

Apenas 55\% das mulheres avaliadas realizaram mamografia previamente, evidenciando possíveis falhas no acesso ao rastreamento e consequente retardo no diagnóstico do câncer de mama nessa região. A prevalência de mulheres que já haviam realizado mamografia foi bem maior no grupo de mulheres residentes em local com mamógrafo disponível (73,6\%), em relação àquelas moradoras nos municípios sem acesso ao exame $(52,1 \%)$. Os índices reduzidos chamam a atenção para a necessidade de implementação do acesso dessas mulheres às orientações e condutas de prevenção e rastreamento do câncer de 
mama, especialmente à mamografia. Portanto, os achados observados indicam a importância da busca ativa de pacientes para rastreamento do câncer mamário em locais onde mamógrafos não são disponibilizados.

Ao comparar mulheres atendidas pelos setores público e privado, Marchi et al. 13 encontraram que $45,8 \%$ das mulheres que foram atendidas nos serviços públicos já haviam realizado mamografia previamente, contra $79,5 \%$ das usuárias de serviços privados. Os motivos para a não realização da mamografia são diversos, podendo variar de falta de recurso financeiro, medo, ausência de equipamento próximo ao local de moradia, dificuldade na marcação do exame por meio do SUS, não ter tido acesso à consulta, achar o exame desnecessário, até simples esquecimento 11 . Entretanto, maior renda familiar, maior escolaridade e moradia na zona urbana, assim como residir fora das regiões Norte e Nordeste do país, estão associadas a uma maior prevalência de realização de exames mamográficos 9 .

$\mathrm{O}$ autoexame de mamas possui custo desprezível, pode ser realizado regularmente, e apresenta relevância na detecção de lesões palpáveis. Além disso, aceitabilidade do teste pelas pacientes, segurança e simplicidade são características importantes desse exame 17,18. Amorim et al. 19 preconizam que a ambientação da mulher com a rotina do autoexame apresenta associação com a realização de mamografia. Entretanto, nossos dados são conflitantes. Apesar de praticamente metade da amostra não ter realizado mamografia prévia, 80,8\% já tinham realizado autoexame das mamas, demonstrando preocupação dessas mulheres com sua saúde.

Essa relação inversa entre a prática de autoexame e mamografia pode ser explicada pelos seguintes fatores: temor em detectar anormalidades, dificuldades culturais e sexológicas, e descrédito na capacidade da detecção da doença em virtude de uma supervalorização do médico e do exame radiológico 14 . Contudo, independentemente da forma como o câncer é detectado, é consenso que sua descoberta precoce torna o tratamento mais eficaz 20 .

Os serviços privados são responsáveis pela maioria dos exames realizados 19 . Tais serviços abrangem, principalmente, a área urbana dos municípios, fazendo com que a população rural em sua maioria seja atendida pelo setor público, que muitas vezes não consegue atuar de forma a atender a demanda necessária.

Estudos já evidenciaram a importância do rastreamento do câncer de mama, assim como a disponibilidade de exames e informação para a população feminina. A Sociedade Brasileira de Mastologia defende a triagem mamográfica anual a partir dos 40 anos, em intervalos de no máximo dois anos entre 50 e 69 anos e, de acordo com a expectativa de vida da mulher, após os 70 anos de idade 21 .

O aumento da mortalidade por esse tipo de câncer no Brasil ocorre em virtude do retardo no diagnóstico na instituição de terapêutica oportuna, fazendo com que as taxas de mortalidade sejam superiores a de países desenvolvidos. Em determinados locais, principalmente os afastados da área urbana, a falta de equipamentos pode fazer com que mulheres dessa região acabem por sobrecarregar hospitais públicos de outras cidades.

Nosso estudo, utilizando o mamomóvel, identificou 37 mulheres com câncer de mama que foram imediatamente encaminhadas para tratamento. Esses casos teriam seu diagnóstico retardado, com consequências para a taxa de cura, caso não houvesse a intervenção do mamomóvel.

Em 2005, a razão entre o número de mamógrafos no Brasil por 100 mil habitantes era de 1,8, sendo que a grande maioria destes aparelhos está localizada nos grandes centros urbanos (Instituto de Comunicação e Informação Científica e Tecnológica em Saúde, Fundação Oswaldo Cruz. Avaliação do Desempenho do Sistema de Saúde. http://www.proadess.icict.fiocruz.br, acessado em 02/Set/2013). Há municípios no Rio Grande do Sul onde as mulheres necessitam se deslocar por distâncias de até $500 \mathrm{~km}$ para realizar mamografia. A associação entre a maior distância até o mamógrafo mais próximo e a não realização de mamografia prévia pôde ser constatada em nosso estudo. É possível que essa situação, de falta de aparelhos especializados para o diagnóstico de câncer de mama, possa ser ainda mais marcada em outras regiões mais pobres do país. Nesse sentido, a intervenção proposta por nosso trabalho demonstra que a disponibilização de exames é necessária na prevenção do câncer de mama, principalmente em locais onde tal prática não é comum. Quase metade das mulheres de nossa amostra nunca havia realizado mamografia anteriormente, demonstrando que a intervenção com a unidade móvel levou informação e, sobretudo, determinou a inclusão dessas mulheres no grupo de rastreamento do câncer mamário.

Portanto, os resultados do nosso estudo demostram que não há equidade no acesso ao rastreamento do câncer mamário, confrontando princípios básicos de universalidade do SUS, assegurados na Constituição Brasileira. Além disso, nossa intervenção permite concluir que o rastreamento ativo utilizando unidade móvel pode ser uma alternativa viável e altamente resolutiva, melhorando significativamente não só o acesso 
à mamografia, mas, a realização de diagnósticos mais precoces e, consequentemente, otimizando o início do tratamento do câncer de mama, com impacto importante na redução da mortalidade pela doença.

\section{Resumen}

El objetivo fue evaluar un programa de prevención y diagnóstico precoz del cáncer de mama, realizado con un equipo móvil de mamografía que visitó ciudades de Río Grande do Sul, el estado más austral en Brasil, donde estos exámenes no estaban disponibles. Se compararon las características de esas mujeres con las de mujeres que fueron remitidas a mamografía en la ciudad de Pelotas, donde ese equipo tiene amplia disponibilidad. Con la unidad móvil fueron examinadas 8.607 mujeres en 33 municipios, se identificaron 37 casos de cáncer de mama, que corresponden a 4,7 casos por 1.000 exámenes. En Pelotas, 1.312 pacientes se sometieron a la mamografía, y la prevalencia del cáncer de mama era de 6,9 casos por 1.000 exámenes. No hubo diferencias estadísticamente significativas entre las dos prevalencias, aun después de ajuste para posibles factores de confusión. La intervención demuestra que la disponibilidad de las pruebas es necesaria para la prevención y diagnóstico precoz, así como para determinar la identificación de los casos, cuyo diagnóstico sufriría atrasos, si este servicio no estuviera activo.

Mamografía; Tecnología Biomédica; Neoplasias de la Mama

\section{Colaboradores}

D. V. Renck participou na concepção do projeto do artigo, levantamento de dados, revisão crítica, redação e aprovação da versão final. F. Barros e M. L. Sclowitz colaboraram na revisão crítica, redação e aprovação da versão final. M. R. Domingues contribuiu na análise dos dados, revisão crítica, redação e aprovação da versão final. M. C. Gonzalez colaborou na análise estatística, revisão crítica, redação e aprovação da versão final. E. L. Caputo participou na redação e aprovação da versão final. L. M. Gomes contribuiu na revisão crítica e aprovação da versão final. 


\section{Referências}

1. Breen N, Wagener D, Brown M, Davis W, BallardBarbash R. Progress in cancer screening over a decade: results of cancer screening from the 1987 , 1992, and 1998 National Health Interview Surveys. J Natl Cancer Inst 2001; 93:1704-13.

2. Godinho ER, Koch HA. Rastreamento do câncer de mama: aspectos relacionados ao médico. Radiol Bras 2004; 37:91-9.

3. Anderson O, Shyyan R, Eniu A, Smith R, Yip C, Bese $\mathrm{N}$, et al. Breast cancer in limited-resource countries: an overview of the Breast Health Global Initiative 2005 guidelines. Breast J 2006; 12 Suppl 1:S3-15.

4. Martins E, Freitas-Júnior R, Curado MP, Freitas NMA, Oliveira JC, Silva CMB. Evolução temporal dos estádios do câncer de mama ao diagnóstico em um registro de base populacional no Brasil Central. Rev Bras Ginecol Obstet 2009; 31:219-23.

5. Instituto Nacional de Câncer. Controle do câncer de mama: documento de consenso. http://www. inca.gov.br/publicacoes/Consensointegra.pdf (acessado em 20/Nov/2012).

6. Robles S, Galanis E. Breast cancer in Latin America and the Caribbean. Rev Panam Salud Pública 2002; 11:178-85.

7. Adams E, Florence C, Thorpe K, Becker E, Joski P. Preventive care: female cancer screening 19962000. Am J Prev Med 2003; 25:301-7.

8. Thuler LC. Considerações sobre a prevenção do câncer de mama feminino. Rev Bras Cancerol 2003; 49:227-38.

9. Lima-Costa M, Matos D. Prevalência e fatores associados à realização da mamografia na faixa etária de 50-69 anos: um estudo baseado na Pesquisa Nacional por Amostra de Domicílios (2003). Cad Saúde Pública 2007; 23:1665-73.

10. Instituto Nacional de Câncer. Incidência de câncer no Brasil: estimativa 2012. Rio de Janeiro: Instituto Nacional de Câncer; 2011.

11. Godinho E, Koch H. O perfil da mulher que se submete a mamografia em Goiânia: uma contribuição a "bases para um programa de detecção precoce do câncer de mama”. Radiol Bras 2002; 35:139-45.
12. Humphrey L, Helfand M, Chan B, Woolf S. Breast cancer screening: a summary of the evidence for the U.S. Preventive Services Task Force. Ann Intern Med 2002; 137(5 Part 1):347-60.

13. Marchi A, Gurgel M, Fonsechi-Carvasan G. Rastreamento mamográfico do câncer de mama em serviços de saúde públicos e privados. Rev Bras Ginecol Obstet 2006; 28:214-9.

14. Sclowitz M, Menezes A, Gigante D, Tessaro S. Condutas na prevenção secundária do câncer de mama e fatores associados. Rev Saúde Pública 2005 39:340-9.

15. Souza R, Lazzaron A, Defferrari R, Borba A, Scherer L, Frasson A. História familiar em segundo grau como fator de risco para câncer de mama. Rev Bras Ginecol Obstet 1998; 20:469-73.

16. Dias-da-Costa JS, Olinto MTA, Bassani D, Marchionatti CRE, Bairros FS, Oliveira MLP, et al. Desigualdades na realização do exame clínico de mama em São Leopoldo, Rio Grande do Sul, Brasil. Cad Saúde Pública 2007; 23:1603-12.

17. Borba A, Souza RD, Lazzaron A, Defferrari R, Scherer L, Frasson A. Frequência de realização e acurácia do auto-exame das mamas na detecção de nódulos em mulheres submetidas à mamografia. Rev Bras Ginecol Obstet 1998; 20:37-43.

18. Davim RMB, Torres GDV, Cabral MLN, Lima VM, Souza MA. Auto-exame de mama: conhecimento de usuárias atendidas no ambulatório de uma maternidade escola. Rev Latinoam Enferm 2003; 11:21-7.

19. Amorim V, Barros M, César C, Carandina L, Goldbaum M. Fatores associados a não realização da mamografia e do exame clínico das mamas: um estudo de base populacional em Campinas, São Paulo, Brasil. Cad Saúde Pública 2008; 24:2623-32.

20. Stein AT, Zelmanowicks AM, Zerwes FP, Biazus JVN, Lázaro L, Franco LR. Rastreamento do câncer de mama: recomendações baseadas em evidências. Rev AMRIGS 2009; 53:438-46.

21. Kemp C, Petti D, Ferraro O, Elias S. Câncer de mama: prevenção secundária. Brasília: Associação Médica Brasileira; 2002.

Recebido em 30/Jan/2013

Versão final reapresentada em 12/Mai/2013

Aprovado em 01/Ago/2013 